DOI: http://dx.doi.org/10.1590/1981-7746-sol00264

\title{
Pareceristas ad hoc do volume 17 (ano 2019)
}

Adriana Leão / Universidade Federal do Espírito Santo

Adriana Teixeira Reis / Fundação Oswaldo Cruz

Alberto Durán González / Universidade Estadual de Londrina

Alexandre Favero Bulgarelli / Universidade Federal do Rio Grande do Sul

Álvaro Roberto Crespo Merlo / Universidade Federal do Rio Grande do Sul

Amanda de Lemos Mello / Universidade Federal de Santa Maria

Ana Lucia Ferreira / Universidade Federal do Rio de Janeiro

Ana Paula Alves Ribeiro / Universidade do Estado do Rio de Janeiro

Ana Paula Hemmi / Universidade Federal dos Vales do Jequitinhonha e Mucuri

Angela Oliveira Casanova / Fundação Oswaldo Cruz

Annatália Meneses de Amorim Gomes / Universidade do Ceará

Antônio Augusto Ferreira Carioca / Universidade Federal do Ceará

Átala Lotti Garcia / Universidade Federal do Espírito Santo

Bianca Antunes Cortes / Fundação Oswaldo Cruz

Brigida Gimenez Carvalho / Universidade Estadual de Londrina

Camila Teixeira Heleno de Araújo / Universidade Federal de Minas Gerais

Carla Rosane Paz Arruda Teo / Rede Metodista de Educação

Catarina Dahl / Universidade Federal do Rio de Janeiro

Cibele Bossle / Universidade Federal do Rio Grande do Sul

Cílson César Fagiani / Universidade de Uberaba

Claudia Tartaglia Reis / Prefeitura Municipal de Saúde de Cataguazes

Cláudia Valente Cavalcante / Pontifícia Universidade Católica de Goiás

Claudia Valéria Cardim da Silva / Universidade do Estado do Rio de Janeiro

Cláudio Fortes Garcia Lorenzo / Universidade de Brasília

Cleonice Andréa Alves Cavalcante / Universidade Federal do Rio Grande do Norte 
Cynthia Maria Barboza do Nascimento / Universidade Federal de Pernambuco Daniela Dellagrave / Grupo Hospitalar Conceição

Denise Yoshie Niy / Ministério da Saúde

Denize Cristina de Oliveira / Universidade do Estado do Rio de Janeiro

Edu Cavadinha / Universidade de Brasilia

Eduardo de Paula Lima / Universidade Federal de Minas Gerais

Eduardo Melo / Fundação Oswaldo Cruz

Elida Azevedo Hennington / Fundação Oswaldo Cruz

Elisete Casotti / Universidade Federal do Rio De Janeiro

Erika Rodrigues De Almeida / Ministério da Saúde

Eronildo Felisberto / Instituto de Medicina Integral Prof. Fernando Figueira

Ester Massae Okamoto Dalla Costa / Universidade Estadual de Londrina

Felipe Proenço de Oliveira / Universidade Federal da Paraíba

Fellipe Coelho Lima / Universidade Federal do Rio Grande do Norte

Fernando Donato Vasconcelos / Ministério do Trabalho

Fernando Martins Carvalho / Universidade Federal da Bahia

Filippina Chinelli / Fundação Oswaldo Cruz

Flavia Carotta / Secretaria de Estado da Saúde de São Paulo

Francis Sodre / Universidade Federal do Espirito Santo

Francisco Arnoldo De Miranda / Universidade Federal do Rio Grande do Norte

Gisele O’Dwyer De Oliveira / Escola Nacional de Saude Pública Sergio Arouca

Guilherme Souza Cavalcanti de Albuquerque / Universidade Federal do Paraná

Henrique Santanna Dias / Secretaria de Estado de Saúde do Rio de Janeiro

Iale Falleiros Braga / Escola Politécnica de Saude Joaquim Venâncio

Inacia Sátiro Xavier de França / Universidade Estadual da Paraíba

Isabela Soares Santos / Fundação Oswaldo Cruz 
Ivana Cristina de Holanda Cunha Barreto / Universidade Federal do Ceará

João Andrade Sales / Universidade Federal do Rio de Janeiro

Jorge Tarcisio Falcão / Universidade Federal do Rio Grande do Norte

José Luiz Alcantara Filho / Universidade Federal Fluminense

Jose Roque Junges / Universidade do Vale do Rio dos Sinos

Karina Araújo Pinto / Universidade Federal da Bahia

Katia Elizabeth Puente-Palacios / Universidade de Brasília

Leda Aparecida Vanelli Nabuco de Gouvêa / Universidade Estadual do Oeste do Paraná

Leonardo de Magalhães Leite / Universidade Federal Fluminense

Letícia Batista da Silva / Fundação Oswaldo Cruz

Lígia Amparo da Silva Santos / Unversidade Federal da Bahia

Lucas Barbosa Pelissari / Instituto Federal de Educação, Ciência e Tecnologia do Sul de Minas Gerais

Lucas Wan Der Maas / Universidade Federal De Minas Gerais

Luciana Santos Dubeux / Instituto de Medicina Integral Professor Fernando Figueira

Lucieli Dias Pedreschi Chaves / Universidade de São Paulo

Manoel Porto Junior / Instituto Federal Sul-Rio-Grandense

Manoela De Carvalho / Universidade Estadual do Oeste do Paraná

Mara Alice Conti Takahashi / Centro de Referência em Saúde do Trabalhador Piracicaba

Marcelo de Castro Meneghim / Universidade Estadual de Campinas

Maria Aparecida Vieira / Universidade Estadual de Montes Claros

Maria Elizabeth Antunes Lima / Universidade Federal de Minas Gerais

Maria Henriqueta Luce Kruse / Universidade Federal do Rio Grande do Sul

Maria Inês Carsalade Martins / Fundação Oswaldo Cruz

Maria Nivalda De Carvalho-Freitas / Universidade Federal de São João Del Rei

Mariana Bertol Leal / Ministério da Saúde

Marina Pereira Rezende / Universidade do Triângulo Mineiro 
Mário Luiz Ferrari Nunes / Universidade Estadual de Campinas

Maristela Dalbello-Araujo / Universidade do Estado do Espírito Santo

Maurício Roberto Campelo De Macedo / Universidade Federal do Rio Grande do Norte

Mayla Cosmo Monteiro / Pontifícia Univesidade Católica Rio de Janeiro

Monica Lima / Universidade Federal da Bahia

Mônica Martins De Oliveira / Universidade Estadual de Campinas

Monica Vieira / Funcação Oswaldo Cruz

Murilo Mariano Vilaça / Fundação Oswaldo Cruz

Nilton Pereira Junior / Universidade Federal de Uberlândia.

Paulo Roberto Haidamus Bastos / Universidade Federal de Mato Grosso do Sul

Paulo Roberto Vasconcellos da Silva / Fundação Oswaldo Cruz

Pedro Renan De Oliveira / Universidade Estadual do Ceará

Priscila Neves Silva / Fundação Oswaldo Cruz

Regina Célia Gollner Zeitoune / Universidade Federal do Rio de Janeiro

Robson da Fonseca Neves / Universidade Federal Da Paraíba

Ronaldo Teodoro Dos Santos / Universidade do Estado do Rio de Janeiro

Rondineli Mendes da Silva / Fundação Oswaldo Cruz

Rosa Maria Castilhos Fernandes / Centro Universitário La Salle

Rosane Fontana / Universidade Regional Integrada do Alto Uruguai

Rosemeire Aparecida Scopinho / Universidade Federal de São Carlos

Simone Santos Oliveira / Fundação Oswaldo Cruz

Sueli Miyuki Yamauti / Universidade de Sorocaba

Tatiana Landini / Universidade Federal de São Paulo

Tatiana Lionço / Universidade de Brasília

Thaís de Souza Lapa / Universidade Estadual de Campinas

Thiago Dias Sarti / Universidade Federal do Espirito Santo 
Túlio Batista Franco / Universidade Federal Fluminense

Tulio Romerio Quirino / Universidade Federal de Pernambuco

Valdir De Castro Oliveira / Universidade Federal de Minas Gerais

Valéria Marli Leonello / Universidade de São Paulo

Valquiria Farias Bezerra Barbosa / Instituto Federal de Educação Ciência e Tecnologia de Pernambuco Valquíria Padilha / Universidade de São Paulo

Vandeleia Pulga / Hospital Conceição

Vera Lucia Felicetti / Centro Universitário La Salle

Vera Navarro / Universidade de São Paulo

Vinício Oliveira Da Silva / Universidade Federal do Paraná

Vladimir Andrei Arce / Universidade Federal da Bahia 